ARTIGOS

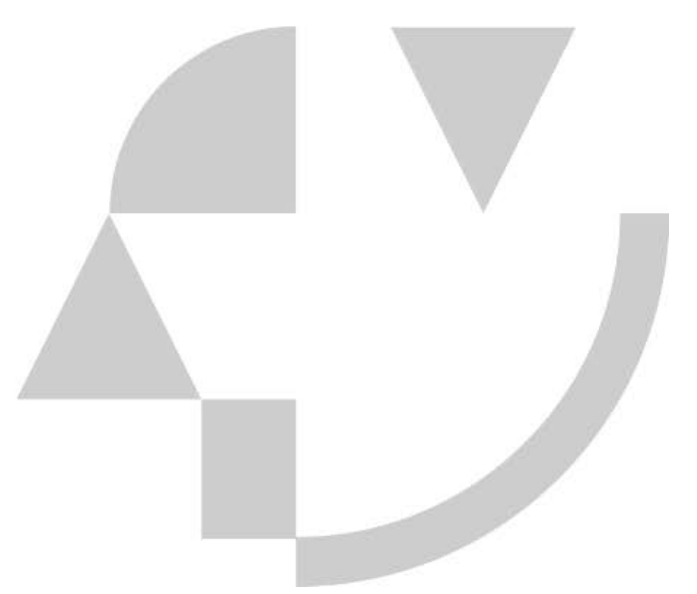





\title{
pro.posições
}

\section{Formación de maestros: reflexiones desde la didáctica y el cuerpo subjetivo (motricidad)}

\section{Teacher formation: reflection from the curriculum and subjective body (body leib)}

Jose Ayala Zuluaga(i) (ii)

Samuel de Souza Neto(iii)

(i)Universidad del Quindío, Educación, Armenia, Quindío, Colombia.

(ii)Universidade Estadual Paulista (Unesp), Educação, Rio Claro, SP, Brasil.

jeayalazu@uniquindio.edu.co

(iii)Universidade Estadual Paulista (Unesp), Educação, Rio Claro, SP, Brasil. samuelsn@rc.unesp.br

\begin{abstract}
Resumen
En el siguiente ensayo se ubican algunas consideraciones de lo educativo en Latinoamérica, los sueños y las emergencias que se develan a partir de lo que está aconteciendo en este escenario. Partiendo de allí, se exponen consideraciones sobre la formación de maestros y el tránsito entre el modelo técnico y el modelos reflexivo, en tal cuestión se soporta la concepción de didáctica y como esta, en su avance teórico y experimental está sujeta al modelo cognitivo de racionalidad técnica. También se hacen apreciaciones sobre lo denominado como cuerpo subjetivo y las posibles consideraciones para aportar en la didáctica y la formación del maestro si se está pensando en procesos educativos identitarios desde lo latinoamericano.

Palabras clave: formación de maestros, didáctica, motricidad
\end{abstract}




\section{pro.posições}

ISSN 1980-6248

\section{Abstract:}

In the following essay some of the educational realities in Latin America, dreams and emergencies that are revealed from what is happening in this scenario, starting from there are located, some considerations on the teachers of formation and their path is exposed between the technical model and reflective model in the design of such an issue is supported teaching and how this, in its theoretical and experimental progress is subject to the cognitive model of technical rationality. Assessments of what termed as subjective body (body leib) and make possible considerations for both teaching and teacher formation if you are thinking of identity from the Latin American educational processes are also made.

Keywords: teacher formation, teaching, body leib

\section{Un escenario de reflexión}

En la actualidad es innegable aquello de la necesidad del desarrollo, para las culturas y sociedades, se considera central avanzar en procesos de lo que consideran desarrollo. Para los países latinoamericanos esta situación no es ajena y mucho más, cuando la mayoría (si no todos) de ellos son considerados países en desarrollo. Lo político, lo religioso, lo económico entre otros aspectos, es revisado y planteado constantemente pero quizás el de mayor pronunciación por todos los estamentos, es la educación.

La ONU (2012), la OEI (2013), los planes de desarrollo de países como Brasil, Argentina, Colombia, Chile; autores como Alarcão (1996), Beillerot (2003), Cajiao (2004), Lüdke (2003), Valencia (2003), Zuluaga (2006) hacen un llamado por la pronta revisión de los procesos que piensan, planean y constituyen la educación presente y del futuro en Latinoamérica. Allí, un punto que puede considerarse de acuerdo común, es la formación de maestros.

Si bien los elementos múltiples de la educación deben ser abordados y trabajados como una trama compleja que se desarrolla a partir de los puntos de acuerdo y sueños de las 


\section{pro.posıções}

sociedades, es necesario precisar que la posibilidad de alcanzar dicho proceso se fortalece en la calidad de los maestros que se tengan para llevar a cabo la labor educativa.

Con dicha premisa, entonces, es necesario pensar en los acontecimientos que rodean la formación de profesores, generalmente estos están matizados por el aprendizaje de teorías en lo pedagógico, lo didáctico, lo metodológico, lo evaluativo y las propias de la disciplina que en algún momento los maestros van a enseñar. Esos saberes pueden ser considerados relevantes y de estudio pormenorizado al momento de investigar sobre la formación del maestro, para los autores de este texto, existe una relevancia en el tema de la didáctica, sobre el sentido de su relación directa con lo que se quiere compartir en el aula y lo que se alcanza (proceso de enseñanza-aprendizaje).

En cuanto a la didáctica, en las últimas décadas existe un avance en lo teórico y experiencial Dominguez (2006), Izquierdo (1997), Comas \& Olson (1998), Tamayo (2010), Tamayo \& Orrego $(2003,2009)$ afirman que en tal sentido, se ha adquirido una especie de estatuto disciplinar, metodológico y epistemológico que lo diferencia de la pedagogía. Las búsquedas por parte de la comunidad académica que se preocupa por la enseñanza sigue su ritmo y alcance generoso, de igual manera en ella se observa una centralidad en la construcción que se soporta en lo "cognitivo".

En consonancia con lo anterior, Ayala (2010, 2012, 2013) menciona los soportes y "fortalezas" en lo cognitivo de la didáctica, y precisa la necesidad de considerar si las perspectivas didácticas no se deben "abrir" a otras posibilidades, que consideren algunas características que no estén ancladas solo en lo cognitivo, es decir, transitar hacia las búsquedas de una didáctica con otras esferas de acción e intencionalidad.

Una opción está soportada en aquello que se denomina desde la fenomenología el cuerpo subjetivo (cuerpo Leib). La enseñanza tiene en tal sentido, un arraigo en lo que se genera desde aristas experienciales que pueden contribuir de otra manera a su fortalecimiento y desempeño en los procesos.

Es así como desde una perspectiva corporal de la didáctica se asume que un buen escenario de discusión para esta trama es la formación de maestros en general. Se considera de esta manera, ya que por diversas cuestiones el trabajo con el cuerpo de estos profesionales hace que lo referido (didáctica con características corporales-experienciales) contenga una connotación amplia por su mismo quehacer. Los profesores desde su bagaje motriz tienen un 


\section{pro.posições}

"discurso" de vida que puede acompañar estas reflexiones y concentrar el escenario de una propuesta.

Con los puntos de la situación problema expuestos, aquí se expresan algunas consideraciones que aportan en la relación formación de maestros, didáctica y cuerpo subjetivo, ya que en las búsquedas del conocimiento, es necesario visibilizar otras formas de trabajo que pueden estar acordes con las miradas del pensamiento y realidad Latinoamericana.

\section{Formación de maestros entre el modelo técnico y el modelo reflexivo}

Viene sucediendo en Latinoamérica (Brasil, 1990; Ecuador, 2007; Argentina, 2010; Colombia, 2014; México, 1999; Nicaragua, 2009) que los gobiernos lanzan programas que piensan en el desarrollo, allí se suscriben procesos como la innovación, el desarrollo tecnológico, el fortalecimiento empresarial, el aumento del empleo, de la salud entre otros factores. En estos aparece la educación como la base que soporta, a largo plazo, que los factores mencionados se logren; se menciona que en tal sentido, debe priorizarse la formación de maestros. Lo anterior se entiende en la medida que son procesos longitudinales y que para sujetos críticos, reflexivos, autónomos y con conciencia social, también es necesario que quien oriente estos procesos tenga unos niveles de calidad y asertividad para llegar el alcance de los objetivos que la sociedad se propone.

En Latinoamérica se viene gestando desde la década del setenta del siglo XX un movimiento educativo-pedagógico (Alarcão, 1996; Arbeusi \& Rueda, 2003; Hernández, 2007; Schön, 1992; Souza, 2001) que anuncia la importancia de la formación de maestros. Se concibe a los educadores como fundamentales en la transformación del contexto. Al respecto, la educación debe responder por un profesional altamente capacitado, que contribuya al cambio social a través de su quehacer (Hernández, 2007, p.15).

Pensando lo anterior, se han implementado modelos de formación de maestros en pro de contribuir en la solución de las problemáticas sociales y se han logrado avances. Latinoamérica es una sociedad más avanzada que hace 40 años, sin embargo, también es necesario pensar y mencionar, que los procesos de sujetos y sociedades críticas, autónomas, 


\section{pro.posıções}

reflexivas están muy lejos de alcanzarse (Ayala, 2013; Díaz, 2009; Souza, Cerignoni, \& Gomes, 2010), producto de ello, se han mencionado los Modelos de formación profesional, ciencia aplicada y reflexivo (Amaral, Moreira \& Ribeiro, 1996; Pimienta \& Ghedin, 2002; Schön, 1995).

En primera instancia se precisa considerar que el modelo de formación profesional o de maestría, si bien tiene perspectivas epistemológicas que lo ubican como un proceso amplio, su aplicación lo ha llevado a ser referido como un trabajo de imitación de técnicas, en el que el profesor impregna en los estudiantes lo que considera pertinente (Amaral et al., 1996; Schön, 1995), en este, los retos educativos generalmente son una imposición desde el saber sabio (profesor) hacia el saber aprendido (estudiante). Es una formación que se repite en el tiempo, si los futuros docentes aprenden de esta manera, se puede pensar que de esa misma forma enseñarán y constituirán sus procesos educativos desde esta lógica.

En segunda instancia se tiene el modelo de ciencia aplicada o racionalidad técnica, aquí se busca que el profesor desde la resolución de problemas de enseñanza aplique investigación y se mueva en las lógicas de la ciencia positivista, las críticas a dicho modelo pasan por el hecho de que su aplicación ha decantado en ejecuciones de orden instrumental que hacen "ver" las situaciones educativas como repetitivas (Amaral et al. 1996; Pimienta \& Ghedin, 2002). Se puede mencionar que este modelo tiene su "aceptación” en la inmadurez del desarrollo del conocimiento en la formación de maestros, debido a que se observa una aplicación en el estricto sentido de las ciencias experimentales en un campo de conocimiento que dista de dicha lógica, en el sentido de que quiere resolver las cuestiones epistemológicas, metodológicas, evaluativas de la formación de maestros desde la racionalidad técnica.

El tercer modelo en formación de maestros es el denominado reflexivo, en este se busca que las personas incluidas actúen de forma autónoma, logrando identificar y resolver problemas, planificar estrategias, ubicar divergencias, ser múltiples en las soluciones, pero ante todo, reflexionar sobre la realidad educativa estudiada en consonancia con contribuir de manera positiva a la comunidad (Amaral et al., 1996; Beillerot, 2013; Ludke, 2013; Pimienta \& Ghedin, 2002; Schön, 1992, 1995 ). Se puede decir, este modelo está en sintonía con ese sueño latinoamericano, de la emancipación, la formación de maestros tiene en este, una línea de compromiso social ante las realidades de la comunidad. 


\section{pro.posições}

Con respecto a los modelos se pueden discutir muchos aspectos, pero uno relevante es que si bien en la formación de maestros los discursos están en consonancia con lo que se plantea en el modelo reflexivo, parece que las realidades de formación y actuación de los profesores están apalancadas en los dos primeros (Formación profesional y ciencia aplicada). Se asume esta consideración ya que los procesos educativos y los resultados de los estudiantes siguen una línea de comportamiento hacia los dos modelos mencionados, es decir, los avances educativos en Latinoamérica no parecen estar cercanos a las exigencias y sueños del modelo reflexivo.

Bajo la lente de que las realidades y los sueños son elementos distantes los unos de los otros, Zuluaga (2003, p.31-35) y Zaambello, Barreto y Correa (2010, p.63) hacen críticas relacionadas con la formación de profesores que dan cuenta de una acentuación de modelos estáticos y cerrados, y mencionan cómo el excesivo atomismo de los conceptos y las prácticas de enseñanza permiten manifestar un "enrarecimiento de la pedagogía"; es así como se viene influyendo negativamente en los procesos educativos que atiendan a un proyecto de país.

Ante la incoherencia entre lo que se desea, lo que se observa y lo que se hace, se asumen algunos factores principales que son causantes de este enrarecimiento, tales como: 1) Ausencia de un objeto común. Referido a que la enseñanza debe ser central en la formación de maestros y esta no es considerada así. 2) La práctica docente o el simulacro de práctica. Las instituciones formadoras de maestros no se preocupan porque el acercamiento profesional de los futuros maestros sea de calidad. 3) La imposibilidad del maestro de pensarse. Traída de la postura romántica de la pedagogía en la cual se menciona que el único sujeto importante del proceso de la educación es el niño aprendiz. 4) Las tendencias profesionalizantes. Los maestros son considerados como sujetos que el mercado laboral necesita. 5) La falta de articulación entre los contenidos específicos de cada área y los contenidos pedagógicos. 6) Los proyectos pedagógicos no se sustentan en la realidad de la escuela y que, no siempre, guardan relación con lo cotidiano del curso de licenciado. Y 7) El distanciamiento entre el saber pedagógico teórico y la actividad práctica del salón de clases. (Gallego, 1999; Pimienta \& Ghedin, 2002; Schön, 1992, 1995; Zambello et al., 2010; Zuluaga, 2003 )

Considerando las situaciones de la racionalidad técnica y el repeticionismo en la educación y como estas pueden relacionarse con muchos eventos, uno que se visibiliza de manera relevante en este texto, son las historicidades que se han acumulado a lo largo de 


\section{pro.posições}

periodos en el cuerpo, que el sujeto maestro ha "encarnado" y es visto como mecánico e instrumental, donde las prácticas están sumergidas en dicha situación. El cuerpo y su acción repiten, modelan en un solo sentido.

Ayala (2013), Cyrino (2012), Jaramillo (2008) y Murcia (2003), mencionan cómo la formación de maestros en educación física no ha dejado de lado aquellas tradiciones que consideran al sujeto como bio-fisico funcional con exclusividad. Al realizar estudios de orden comprensivo, se detectó que uno de los factores comunes que influyen sobre las prácticas de los maestros está soportado en procesos de formación docente cerrados e inflexibles. Estas posiciones permiten pensar sobre el papel y la real influencia de la formación de maestros en la sociedad y en las disciplinas, a su vez, formular la pregunta por la inclusión de las alternativas del conocimiento y el alcance que debe tener un maestro para ejercer su profesión con calidad.

\section{Aperturas desde la didáctica}

Son evidentes los avances teóricos y prácticos sobre la enseñanza (esta entendida como el eje de la didáctica, Auduriz-Bravo, 2001; Dominguez, 2006) ${ }^{1}$ que se han dado en los últimos tiempos, este proceso está marcado por elementos epistémicos complejos, que permiten hoy, tener un objeto de estudio que es la enseñanza y en tanto ser considerada como una teoría de la didáctica.

Mallart (2000, p.5-14) sostiene que etimológicamente, el término Didáctica procede del griego: didaktiké, didaskein, didaskalia, didaktikos, didasko. Estos términos tienen relación con el verbo enseñar, instruir, exponer con claridad. Este autor afirma que desde las propuestas de Comenio, utilizaron la denominación de Didáctica tomada del latín, no del griego. Para Comenio (1986), en su obra Didáctica Magna, esta era “el artificio universal para enseñar todas las cosas a todos, con rapidez, alegría y eficacia” (p.197).

\footnotetext{
${ }^{1}$ En Algunos escenarios se discute primero sobre la separación o no de la enseñanza y el aprendizaje, aquí se tomará posición sobre el objeto de estudio que hoy debe desarrollarse desde la enseñanza. Y el segundo caso, es si la enseñanza es el objeto de estudio de la didáctica y las relaciones teóricas que allí se construyen, se defienden las creaciones que se dan en estas. Estos no serán discutidos en el artículo, para tal efecto sugerimos la lectura de Auduriz-Bravo (2001); Dominguez (2006); Garrido (2006); Tamayo y Orrego, (2009).
} 


\section{pro.posições}

Hoy, el término enseñanza está completamente extendido en el ámbito europeo continental y países de su órbita cultural. En Alemania, Francia, Italia, España e Iberoamérica goza de una gran tradición y desarrollo. Pertenece al léxico culto generalizado. Al mismo tiempo hay que destacar que el término es poco usado en todo el territorio anglosajón, aunque no así su contenido. Al mismo contenido se le aplica el nombre de enseñanza o el de aprendizaje, según el punto de vista. Y hoy tiende a coincidir, por una superposición del campo abarcado, con el término currículum (Apple, 1996; Posner, 1999). En la actualidad, autores como Labbé (2007) consideran que la didáctica

es la disciplina científico-pedagógica que tiene como objeto de estudio los procesos y elementos existentes en la enseñanza y el aprendizaje. Es, por tanto, la parte de la pedagogía que se ocupa de los sistemas y métodos prácticos de enseñanza destinados a plasmar en la realidad las directrices de las teorías pedagógicas. (p.93)

La autora infiere que la didáctica está muy vinculada con otras disciplinas pedagógicas como, por ejemplo, la organización escolar y la orientación educativa; la didáctica se propone como el fundamento y regulación de los procesos de enseñanza y aprendizaje.

En una perspectiva diferente a la de Labbé, se asume que la educación en ciencias se preocupa en la actualidad por "enseñar ciencias significantemente” (Tamayo, 2001,). Allí se reúnen algunas características que no sólo están buscando una amalgama para complejizar, sino que se involucran en un estudio sistémico que eleva a nivel de conocimiento científico los avances en didáctica. Dice Tamayo y Orrego (2009, p.13) que se debe ubicar la enseñanza de las ciencias como una disciplina emergente, en relación con diferentes campos del saber y en permanente proceso de diferenciación frente a otras áreas del conocimiento que tradicionalmente han estudiado la problemática de la enseñanza y aprendizaje de las ciencias.

Las relaciones que tiene en la actualidad la didáctica parten de múltiples revisiones y debates en los cuales se manifiesta cómo esta debe hacer uso de varios saberes para lograr su objetivo de formación. Debe describir, analizar y comprender los problemas más relevantes en la enseñanza y el aprendizaje de las ciencias y, diseñar y experimentar modelos que ofrezcan posibles soluciones a la problemática educativa.

Desde esta postura se infiere que la eficacia docente depende de la relación entre lo que se enseña y lo que el estudiante aprende. Es el cambio experimentado por este último, lo que acontece como consecuencia de la actividad del profesor; es un criterio fundamental para 


\section{pro.posições}

determinar el éxito de la actividad didáctica y la relación ideal que ejerce esta como componente científico.

$\mathrm{Al}$ revisar los aportes de los autores sobre la didáctica, términos como lo experimental, su relación con la cientificidad, los apoyos de la teoría neurolingüística, entre otros, "develan" de cierta manera una predilección en lo que podríamos llamar la didáctica centrada en lo cognitivo, en lo que se organiza, desarrolla y tiene su centro en la mente, hecho que lleva a pensar que se repite la imposición del paradigma descartiano y la ubicación de la binaria propia de la racionalidad técnica; si bien es necesario considerar que este proceso efectivamente corresponde a esta tradición, no significa que en la realidad se dé solo de esta manera, se deben buscar otras líneas de fuga que permitan aportes en la enseñanza, es necesario presentar "otras" consideraciones; desde la perspectiva de este artículo, es necesario retomar el carácter existencial (cuerpo subjetivo) para ser referido en la didáctica.

\section{Cuerpo subjetivo y motricidad en clave de educación}

En primera instancia es necesario considerar la inmanencia cuerpo y motricidad. Reconociendo la sinonimia que parece existir por parte de algunos autores sobre el tema (Gallo, 2009; Hurtado, 2008; Kolyniak, 2005), también se asume con autores como Jaramillo (2005) y Planella (2006) que la motricidad de la cual se habla desde las tendencias actuales, no se refiere a esa con contenido exclusivo hacia lo físico, esta puede ser vista con el prisma de la diversidad que permite otras miradas en clave compleja.

En esta manera de ver la motricidad y su relación con el cuerpo subjetivo no se evidencia la tensión entre lo instrumental y trascendente del sujeto a través de su corporeidadmotricidad. Para autores como Arboleda (2009), Gallo (2009), Jaramillo (2005) y Planella (2006), las controversias a nivel epistemológico, vivencial del cuerpo y de la motricidad, radican en orientar y transitar hacia una motricidad que permita ver al sujeto no como un instrumento, sino como un ser complejo y en plenitud para lograr sus anhelos. Esta perspectiva se fundamenta en un ser afectado, y que a su vez lo construye, constituye y es mundo. 


\section{pro.posições}

Para Merleau-Ponty (1991) el “soy cuerpo” se contrapone al “tengo cuerpo”. Planteamiento desde el cual se pretende superar el dualismo cuerpo-mente; e insinuar lo "quiasmático" como la relación equilibrada entre estos dos fenómenos.

El problema principal que preocupa a Merleau-Ponty no es la unión sino la distinción entre conciencia y cuerpo. Esto ha de sostenerse porque el análisis parte de una vivencia en la que el cuerpo ya está unido a la conciencia. Donde el autor concluye que "el cuerpo (motricidad) es nuestro medio general de poseer el mundo". Allí deja un camino expuesto para referirse a lo que se considera como un cuerpo reflexivo, no entendido en términos del cuerpo que piensa, sino en consonancia con ese cuerpo que construye mundo desde la relación equilibrada entre su acción y reflexión.

Desde la perspectiva de este texto, lo anterior se construye en primera instancia desde el concepto de cuerpo Leib o cuerpo subjetivo, entendido como un cuerpo sujeto en el que ocurren acontecimientos, sucesos, hechos, encuentros, desencuentros, realidades; se construyen infinidades de experiencias que permiten pensar ese y otros mundos, que familiarizan al sujeto con su sentido y con su significado vital. Se está hablando así, de un cuerpo que se deja permear y permea, para la mutación, el cambio que logra otras realidades, se entiende ese cuerpo en el sentido de Barbero (2003) que manifiesta: "los sujetos con los que vivimos, especialmente entre las nuevas generaciones, perciben y asumen la relación social en cuanto a una experiencia que pasa básicamente por su sensibilidad y su corporeidad" (p.40). Entonces, el cuerpo significa el espacio para territorios y cultura tanto como agente, promotor, constructor de vida, donde la significación, los procesos sociales, la circulación del mundo propio y ajeno origina la cultura.

Es necesario decir entonces, que existe la posibilidad de estudiar al sujeto desde la experiencia corporal, este hecho se soporta en las circunstancias del sujeto y la relación de movimiento que está atada al cuerpo de la experiencia, es decir, la motricidad le permite al cuerpo Leib, al cuerpo sujeto concebir e incorporar los acontecimientos que construyen y son cultura.

$\mathrm{Y}$ esa motricidad puede ser entendida desde el pensamiento iberoamericano (Arboleda, 2007; Gallo, 2009; Jaramillo \& Trigo, 2005; Kolinyak, 2005; Murcia, 2008 y Sergio, 1985) por ejemplo, Sergio (2006) asume la motricidad como: 


\section{pro.posições}

forma concreta de relacionarse del ser humano con el mundo y con sus semejantes, relación que está caracterizada por intencionalidad y significado fruto de un proceso evolutivo y cuya especificidad, - a su vez, discurren de las relaciones recíprocas entre naturaleza y cultura - por tanto, entre las herencias biológicas y socio-históricas-. La motricidad se refiere a sensaciones conscientes del ser humano en movimiento intencional y significativo, en el espacio- tiempo objetivo y representado, implicando percepción memoria, proyección, afectividad, emoción y raciocinio. (p. 13)

En cuanto a su ejecución o puesta en escena, el autor considera que: "Ese proceso ocurre, de forma dialéctica, en los planos filogenético y ontogenético, expresando y componiendo la totalidad de las múltiples y complejas determinaciones de la continua construcción del hombre” (Sergio, 2005, p. 17).

Desde otras consideraciones sobre motricidad, es necesario referir el trabajo de Benjumea (2011) en relación a la motricidad, en este se explicitan los elementos constitutivos de la motricidad, se realiza un análisis de diversos procesos que confluyen en el tema, aspectos tales como su historia, relaciones teóricas, epistemológicas, metodológicas, ontológicas entre otras, este proceso es un apartado de realce para el tema de la motricidad ${ }^{2}$ desde la perspectiva de Sergio. La autora considera 6 elementos constitutivos en la motricidad ellos son: a) la corporeidad, b) el movimiento, c) la percepción, d) la intencionalidad-consciencia, e) la espacialidad y f) la temporalidad.

Estos constitutivos no son elementos aislados, su interdependencia enfatiza la complejidad de la misma motricidad, dicha consideración conlleva entonces a reflexionar sobre la necesidad de ubicar los trabajos y adelantos en motricidad desde la complejidad y por ende en lo sistémico, donde los aspectos prácticos y teóricos develan nuevas formas y vasos comunicantes para discutir y proponer una mirada diversa, contextualizada y pertinente, se asumen con Benjumea (2011, p. 192) que es un proceso dinámico y constante que permite redes complejas de desarrollo.

Asimismo, Merleau-Ponty (1991), menciona que

\footnotetext{
2 Como se mencionó, la propuesta de Sergio sobre motricidad no se ha agotado en las posturas del autor, sino que en la actualidad existen adelantos por parte de otros autores, en este caso recomiendo revisar la obra de Benjumea (2011) que explicita en forma concreta algunas reflexiones al respecto, o las consideraciones que desde la didáctica ha realizado Toro $(2008,2010)$, asimismo la concepción sobre imaginarios y simbolismo de Murcia (2012).
} 


\section{pro.posições}

la motricidad, tomada en estado puro, ya posee el poder elemental de dar sentido. Aun cuando, más adelante, el pensamiento y la percepción del espacio se liberen de la motricidad y del ser en el espacio, para que podamos representarnos el espacio es preciso que hayamos, primero, sido introducidos en él por nuestro propio cuerpo y que éste nos haya dado el primer modelo de ...."La motricidad es la esfera primaria en donde se engendra, primero, el sentido de todas las significaciones en el dominio del espacio representado. (p. 159).

Lo anterior lleva a considerar y revisar la perspectiva subjetiva de la motricidad; en ella se evidencia un aporte al desarrollo humano, ya que contribuye a diversas dimensiones del ser, lo estético, lo ético y político, entre otros, se logran en y desde la motricidad.

La motricidad en la actualidad puede ser considerada como una ciencia, disciplina o campo del saber en construcción que permite al ser humano encontrarse y potencializarse a sí mismo. Esta alternativa tiene cabida en la medida que permite el accionar para aproximarse, encontrarse y desarrollar las diferentes esferas de la vida.

Considerando lo anterior, la relación cuerpo-motricidad puede ser pensada como una interpretación que procura liberar la modelización fiscalista que existe del cuerpo y su forma de leerlo, es proceso creador que no supone formas fijas, esta relación describe, cuenta, sospecha, deja huellas. Murcia et al. (2005) consideran, en cuanto a la función y los alcances de la motricidad, lo siguiente:

La motricidad ha sido fundamental para alcanzar la condición humana, en tanto ella ha permitido promocionar identidades que definen fortalezas o debilidades de las comunidades, a tal punto de hacerla surgir o desaparecer, las comunidades fueron construyendo un saber sobre el medio que las llevó a adaptar a los recursos para lograr además de la subsistencia, otros desarrollos. (p.38)

La perspectiva subjetiva de la motricidad (cuerpo subjetivo) influye poderosamente en el desarrollo social; por muy especializados que se tornen los usos de la misma, siempre deja develar un elemento social o construcción del medio en el cual se desenvuelve el individuo que hace parte de cualquier manifestación. De esta manera, es necesario referir estas reflexiones hacia el concepto de didáctica y la formación de maestros. 


\section{pro.posições}

\section{Consideraciones que contribuyen en la enseñanza}

Si tal como se expresa en este trabajo, el cuerpo subjetivo es correlato de existencia, si la didáctica tiene otras opciones en las cuales el recorrido vital podría contribuir con otras formas de enseñanza que tengan en cuenta más la totalidad del ser y, si la formación de maestros desde su modelo reflexivo asume al maestro como lector y actor de procesos que tienen en cuenta las exigencias del sujeto y el contexto, entonces, se presume con estas relaciones, que es necesario reflexionar sobre los elementos que entretejen dicha cuestión. Aquí algunas consideraciones.

En Latinoamérica, según lo expuesto, existe un llamado por abordar el modelo reflexivo de maestro en su misma formación, se hace una apuesta por un profesor que esté contextualizado y convergente con las realidades humanas al momento de enseñar. Pero al hacer las revisiones sobre los avances en educación, pareciera que existe una brecha enorme, y en tal sentido, no se considera en este texto que haya un error en lo que se desea pero sí en su desarrollo, que dista de aquello deseado. Se "sospecha" que dicha situación está "aferrada" a la cuestión que en lo discursivo se plantean los elementos teóricos de lo deseado, pero en la realidad, en la práctica reflexiva, no se dan opciones al nuevo maestro de interiorizar dicho proceso, mientras el discurso de formación de maestros latinoamericanos está soportado en las teorías del modelo reflexivo y las didácticas significativas, las acciones que permiten lo anterior, están imitando viejas líneas de formación de profesores.

Puede ser que debido a las preferencias de saberes de orden cognitivo que en los espacios de formación de profesores se da, es común entonces, encontrar procesos en los cuales los nuevos docentes privilegian su enseñanza en la tradición cognitiva. Sería entonces, pensar otras formaciones de profesores, otras didácticas, aunque no se considera imposible que el futuro maestro enseñe en una lógica diferente a la de la prevalencia de la cognición, sí se puede decir que éste le da poca opción a las didácticas con referentes existenciales, ya que lo cognitivo ha sido su formación. Los docentes universitarios, “quizás" deben abandonar sus lógicas de racionalidad técnica y transitar hacia modelos de formación de maestros con mayores y mejores contenidos reflexivos existenciales.

Allí la teorización debe tener cabida, pero debe estar acompañada de formas de enseñanza que "equilibren" lo cognitivo y lo existencial, las didácticas que se construyan pueden estar matizadas con la realidad del aula, y en la lógica que cada sujeto que hace parte 


\section{pro.posições}

del fenómeno de enseñanza, encuentre un escenario para comprender sus encuentros didácticos, los maestros de profesores y estos últimos, deben generar espacios para cohabitar la construcción didáctica.

Aquí se quiere hacer un énfasis, que en lo que llamamos didácticas desde el cuerpo subjetivo en la formación del maestro, y es que no se está realizando un llamado por volver a lo empírico; al reconocer la importancia de la motricidad y la existencia del ser en la formación del maestro, se está hablando de una interrelación entre los elementos reflexivos y la práctica, nos referimos entonces a un "juego" entre las viejas posturas racionalistas y empiristas vistas en clave didáctica, pero a su vez, pensadas cuando se está formando al maestro y el impacto que puedan tener en maestros latinoamericanos que construyen y buscan identidad. 


\section{pro.posıções}

ISSN 1980-6248

\section{Referencias Bibliográficas}

Alarcão, I. (Org.). (1996). Formação reflexiva de professores, estratégias de supervisão. Porto: Editora Porto.

Apple, M. (1996). Discursos sobre educación. México. Ed. Fondo de cultura económica.

Arbesú, I., \& Rueda , M. (2003). La evaluación de la docencia desde la perspectiva del propio docente. Reencuentro. Análisis de problemas universitarios, 36, 56-65.

Arboleda, R. (2009). Las expresiones motrices, una apuesta epistemológica. Medellín-Antioquia: Círculos académicos del instituto universitario de educación física de la universidad de Antioquia.

Arias, J. A. (1975). La antropología fenomenológica de Merleau-Ponty. Madrid: Editorial Fragua.

Auduriz-Bravo, A. (2001). Integración epistemológica en la formación del profesorado de ciencias. Tesis doctoral, Universidad Autónoma de Barcelona, Barcelona.

Barbero, J. (2003). La educación desde la comunicación. Bogotá: Editorial Norma.

Beillerot, J. (2003). A “pesquisa": esboço de uma análise. In M. André, O papel da pesquisa na formação e na prática dos professores. São Paulo: Papirus.

Cajiao, F. (2004). La formación de maestros y su impacto social. Bogotá: Cooperativa Editorial Magisterio.

Comas, W., \& Olson, J. K. (1998). The nature of science in international science education standars documents. In W. F. Mc Comas (Ed.), The nature of science in science education. Rationales and strategies. Dordrecht/Boston/London: Kluwer Academic Publishers.

Comenio, J. A. (1986). Didáctica Magna. Madrid: Editorial Akal.

Cury, C. R. J. (2006). La formación de profesores en Minas Gerais, Brasil. Cambios institucionales de formación docente en curso. In M. R. Campos, \& A. Körner (Coords.), Modelos innovadores en formación de docentes (pp. 127-199). Unesco: Santiago de Chile, Chile. Extractado en enero 13 de 2014, de http://oei.es/docentes/ publicaciones/docentes/modelos_innovadores_formacion_inicial_docente.pdf. 


\section{pro.posıções}

Cyrino, M. (2012). Formação inicial de professores: o compromisso do professor-colaborador e da instituição escolar no processo de estagio supervisionado. Dissertação de Mestrado, Programa de PósGraduação em Educação, Universidade Estadual Paulista, Rio Claro.

Deleuze, G. (2005). Derrames. Entre el capitalismo y la esquizofrenia. Buenos Aires: Cactus.

Diccionario de la Real Academia de la Lengua. (2004a-2012). Microsoft Corporation.

Diccionario Houais da Língua Portuguesa. (2009). (pp. 662-767). Rio de Janeiro: Objetiva.

Dominguez, A. (1998). O ensino: objeto da didáctica. In A. D. Castro, \& A. M. P. Carvalho, Ensinar a ensinar. (pp. 47-63). São Paulo: Thomson Learning Pioneira.

Gallego, R. (1999). Competencias cognoscitivas, un enfoque epistemológico, pedagógico y didáctico. Santa Fe de Bogotá: Editorial Magisterio.

Gallo, L. E. (2009). Aportes antropológico-fenomenológicos sobre la corporalidad y el movimiento bumanos para una educación corporal. Investigación sobre el pensamiento de los principales autores que han influido el discurso de la Educación Física contemporánea en Colombia. Tesis de doctoral, Facultad de Educación, Universidad de Antioquia, Medellín, Colombia.

Hurtado, D. R. (2008, janeiro/abril). Motricidad y corporeidad. Una forma de mirar los saberes del cuerpo. Revista Brasileira de Educação \& Sociedade, 29(102), 119-136 Extractado el dia 15 de marzo de 2009 http://www.cedes.unicamp.br. Enlace: http://www.scielo.br/scielo.php?script=sci_arttext\&pid=S0101- 73302008000100007 $\& \operatorname{lng}=$ es\&nrm $=$ iso\&tlng $=$ es.

Izquierdo, M. (1997). Estructuras retóricas en los libros de texto. Barcelona: Universidad Autónoma de Barcelona.

Jaramillo, L. G. (2007). La complementariedad como posibilidad para investigar en motricidad y desarrollo humano. En Rev: En acción consentido (pp. 124-147). Popayan: Universidad del Cauca..

Jaramillo, L. G. (2008). La ciencia de la motricidad humana: paradigma o paradogma emergente de la Educación Física? Tese de Maestría en Educación. Énfasis en motricidad y aprendizaje, Convenio Universidad del Quindío, Universidad de Caldas. 


\section{pro.posıções}

ISSN 1980-6248

Kolyniak, K. (2005). Propuesta de un glosario inicial para la ciencia de la motricidad humana. In E. Trigo, D. Hurtado, \& L. Jaramillo (Comps.), Con- sentido (pp. 29-37). Popayán: Universidad del Cauca.

Lovisolo, H. (1995). Educación física. A arte da mediação. Rio de Janeiro: Sprint.

Labbé, C. (2007). Diferencias entre didáctica y metodología. En Rev: Alternativa E-ducativa, 293. Extractado el 13 de abril de 2010, http:/ /www.edusal.cl.

Lüdke, M. (2013). A complexa relação entre o professor e a pesquisa. In M. André, O papel da pesquisa na formação e na prática dos professores. São Paulo: Papirus.

Mallart, J. (2000). Desarrollo curricular, organizativo y profesional: una concepción del currículum desde la colaboración del profesorado. In M. Pérez Ferra, Desarrollo curricular. Barcelona: Editorial Paidos

Merleau-Ponty, M. (1991). Fenomenología de la percepción. Barcelona: Planeta Agostino.

Murcia, N. (2003). Los imaginarios de l@s jóvenes ante la clase de Educación Física. Informe final. Armenia: Colciencias; Kinesis.

Organización de las Naciones Unidas - ONU. (2012). La educación que soñamos. Informe del PNUD. Publicado el 31 de Mayo. Extractado el 18 de noviembre de 2013, en: http://www.oei.edu

Pimenta, S. G., \& Ghedin, E. (Orgs.). (2002). Professor reflexivo no Brasil: gênese e crítica de um conceito. São Paulo: Cortez.

Planella, J. (2006). Cuerpo, cultura y educación. Bilbao: Editorial Desclée de Brouwer,

Posner, J. G. (1999). Análisis del currículo (2a ed.). México: Mc. Graw Hill.

Schön, D. (1995). Formar professores como profissionais reflexivos. In A. Nóvoa, (Org.), Os professores e sua formação. Lisboa: Dom Quixote.

Schön, D. (1992). La formación de profesionales reflexivos, hacia un nuevo diseño de la enseñanza y el aprendizaje en las profesiones. Madrid: Paidós; MEC.

Souza Neto, S., Benites, L. C., \& Silva, M. F. G. (2010, dezembro). Da escola de ofício a profissão educação física: a constituição do habitus profissional de professor. Motriæ, 


\section{pro.posıções}

ISSN 1980-6248

16(4). Extractado el 18 de enero de 2014, de http://www.scielo.br/scielo.php?pid=S1980-65742010000400024\&script=sci_arttext.

Souza Neto, S. (2001). Práticas supervisadas em educação física. El papel del supervisor. Ed. Panamericana. São Paulo. Brasil

Tamayo, O. E. (2010). Epistemología de la didáctica de las ciencias. Maestría en Educación, Universidad de Caldas, Manizales.

Tamayo, O. \& Orrego, M. (2003). Caracterización general de la didáctica. Doctorado_en Ciencias de la Educación de Rudecolombia, Cade Universidad de Caldas.

Tamayo, O., \& Orrego, M. (2009) Aportes de la naturaleza de la ciencia y del contenido pedagógico del conocimiento para el campo conceptual de la educación en ciencias. Doctorado en Ciencias de la Educación, Rudecolombia, Manizales.

Valencia, C. (2003). Tendencia pedagógica alemana. Maestría en Educación-Docencia, Universidad de Manizales, Manizales.

Zambello, S., Barreto, J. B., \& Correa, J. R. (2010). A formação de professores e os núcleos de ensino da Unesp. In S. G. de L. Mendonça, R. L. L. Barbosa, \& N. R. Vieira, Núcleos de ensino da Unesp, memórias e trajetórias. São Paulo: Unesp; Cultura Acadêmica.

Zuluaga, O. (2006). De Comenio a Herbart. Educación y Cultura, 17, 45-51.

Submetido à avaliação em 6 de janeiro de 2015; aprovado para publicação em 21 de agosto de 2015. 